\title{
HOLOMORPHIC MAPPINGS OF COMPLEX SPACES
}

\author{
RAGHAVAN NARASIMHAN
}

1. This note deals with some theorems similar to the following theorem announced by $\mathrm{R}$. Remmert [4].

TheOREM. Let $X$ be a holomorphically separable, connected (normal) complex space of dimension $n$. Then, there exists a one-one, holomorphic map of $X$ in $C^{2 n+1}$.

We use throughout the terminology of [2] and [4].

In $\$ 2$, we give a simple proof of the following slightly stronger version of this theorem.

TheOREM 1. Let $X$ be a connected holomorphically separable complex space of dimension $n$, (not necessarily normal). Let $\epsilon>0, K$ a compact set in $X$ and let $\phi_{1}, \cdots, \phi_{2 n+1}$ be arbitrary holomorphic functions on $X$. Then, there is a one-one holomorphic map $f=\left(f_{1}, \cdots, f_{2 n+1}\right)$ of $X$ in $C^{2 n+1}$ such that

$$
\left|f_{i}(x)-\phi_{i}(x)\right|<\epsilon \quad \text { for } x \in K .
$$

In $\S 3$, we consider the existence of holomorphic maps with a given rank on complex spaces countable at infinity. We use the method given here to make a remark on an imbedding theorem for holomorphically complete spaces due to R. Remmert [4].

The author is indebted to Professor H. Cartan for his suggestions and for pointing out that the space of holomorphic functions is complete even for non-normal spaces. The author's thanks are due also to Professor K. Chandrasekharan for his encouragement prior to and during the preparation of this note.

2. We shall denote, throughout this note, the space of holomorphic functions on a complex space $X$, with the compact convergence topology, by $R(X)=R$.

If $X$ is holomorphically separable, it can be shown that $X$ is $K$ complete (in the sense of [1]; we note that it is enough to have, for any $x_{0} \in X$, a holomorphic map $f: X \rightarrow C^{k}$ such that $x_{0}$ is an isolated point of $\left.f^{-1} f\left(x_{0}\right)\right)$, so that, by [1, Satz 8 ], $X$ is countable at infinity. Hence $R(X)$ is metrisable, and by [2, Satz 28], it is complete.

We prove first

THEOREM 2. If $X$ is a connected, holomorphically separable complex space and $\left\{x_{m}\right\}$ a sequence of distinct points of $X$, then there is a holo-

Received by the editors November 23, 1959. 
morphic function $f$ on $X$ such that if $m \neq m^{\prime}$, then $f\left(x_{m}\right) \neq f\left(x_{m^{\prime}}\right) . f$ may be required to approximate an arbitrarily given $\phi \in R(X)$ on a given compact set.

Proof. Let $x, y \in X, x \neq y$. The set $C(x, y)$ of $f \in R$ with $f(x)=f(y)$ is clearly closed in $R$. Also $U=R-C(x, y)$ is dense in $R$; for let $g \in R$. If $g(x) \neq g(y)$, then $g \in U$. If $g(x)=g(y)$ and if $f \in R, f(x) \neq f(y)$, and if $\lambda \neq 0$ then $g+\lambda f \in U$ so that $g \in \bar{U}$; hence $\bar{U}=R$. Thus $C(x, y)$ is nowhere dense in $R$.

Let $C=U_{m \neq m^{\prime}} C\left(x_{m}, x_{m^{\prime}}\right) . C$ is a set of the first category. By Baire's theorem, $R-C$ is dense in $R$. Theorem 2 follows.

We proceed to the proof of Theorem 1. Let $Y=X \times X-\Delta$ ( $\Delta$ being the diagonal of $X \times X)$. Since $X$ is countable at infinity, so is $Y$. Hence any analytic set in $Y$ has at most countably many irreducible components. Let $f_{1} \in R$ satisfy $\left|f_{1}(x)-\phi_{1}(x)\right|<\epsilon, x \in K$, and be nonconstant on any irreducible component of $Y$ (which exists, by Theorem 2). Let $A_{1}$ be the analytic set of points $(x, y) \in Y$ with $f_{1}(x)-f_{1}(y)$ $=0$. Then, clearly, $\operatorname{dim} A_{1} \leqq 2 n-1$. If $A_{1}^{(p)}, p=1,2, \cdots$ are its irreducible components, choose $\left(x_{1}^{p}, y_{1}^{p}\right) \in A_{1}^{(p)}$. By Theorem 2, there is an $f_{2} \in R,\left|f_{2}(x)-\phi_{2}(x)\right|<\epsilon$ on $K, f_{2}\left(x_{1}^{p}\right) \neq f_{2}\left(y_{1}^{p}\right)$. The set $A_{2}$ of $(x, y) \in Y$ with $f_{1}(x)=f_{1}(y), f_{2}(x)=f_{2}(y)$ has $\operatorname{dim} A_{2} \leqq 2 n-2$, for if $B$ is an irreducible component of $A_{2}$ and $B \subset A_{1}^{(p)}$, then $\left(x_{1}^{p}, y_{1}^{p}\right) \in A_{1}^{(p)}$, $\notin B$ so that $\operatorname{dim} B<\operatorname{dim} A_{1}^{(p)} \leqq 2 n-1$. Theorem 1 follows on repeating this process.

3. Let $f$ be a holomorphic map of the purely $n$-dimensional complex space $X, f: X \rightarrow C^{k}$. The rank $r\left(f, x_{0}\right)$ of $f$ at $x_{0} \in X$ is by definition, the codimension at $x_{0}$ of the analytic set $f^{-1} f\left(x_{0}\right)$ (see Remmert [5]).

TheOREM 3. Let $X$ be a purely $m$-dimensional complex space countable at infinity. Suppose that for each $x \in X$ there is a map $X \rightarrow C^{k}$ of rank $\geqq p$ at $x(k=k(x), p$ a fixed integer $>0)$. Then there is an integer $N=N(n)$ and a holomorphic map $X \rightarrow C^{N}$ of rank $\geqq p$ at any point of $X$.

Lemma 1. Let $f=\left(f_{1}, \cdots, f_{k}\right): X \rightarrow C^{k}$ have rank $p$ at $x_{0}$ and $U$ be a neighbourhood of $x_{0}$. Then, there is an $\epsilon>0$ such that if $\phi_{1}, \cdots, \phi_{k}$ $\in R(X)$ and $\left|\phi_{i}-f_{i}\right|<\epsilon$ on $U$, then $\left(\phi_{1}, \cdots, \phi_{k}\right)$ has rank $\geqq p$ at $x_{0}$.

Proof. We may suppose that $X$ is a purely $n$-dimensional analytic set in an open set $G \subset C^{n+m}$. Now, $\operatorname{dim}\left(x_{0}, f^{-1} f\left(x_{0}\right)\right)=n-p$. Hence there is an $m+p$ dimensional analytic plane $Z$ in $G$ through $x_{0}$ such that $x_{0}$ is an isolated point of $Z \cap f^{-1} f\left(x_{0}\right)$. Let $X^{\prime}=X \cap Z$ and $f^{\prime}$ be the restriction of $f$ to $X^{\prime}$. Then $x_{0}$ is an isolated point of $f^{\prime-1} f^{\prime}\left(x_{0}\right)$ 
and so $f^{\prime}$ gives a nowhere degenerate map of a neighbourhood of $x_{0}$ in $X^{\prime}$ by [5, Satz 15]. Hence, by [1, Hilfssatz 3], if $\phi_{i} \in R(X),\left|\phi_{i}-f_{i}\right|$ $<\epsilon$ and if $\epsilon$ is sinall enough, the restriction of $\phi$ to $X$ is nowhere degenerate in a neighbourhood of $x_{0}$ so that $x_{0}$ is an isolated point of $Z \cap \phi^{-1} \phi\left(x_{0}\right)$. Since $\operatorname{dim} Z=m+p, \operatorname{dim}\left(x_{0}, \phi^{-1} \phi\left(x_{0}\right)\right) \leqq n-p$ and Lemma 1 is proved.

Lemma 2. Let $\phi=\left(\phi_{1}, \cdots, \phi_{k}\right): X \rightarrow C^{k}$ have rank $p$ at $x_{0}$ and let $f \in R(X)$. Then, for all constants $\lambda$, excepting finitely many, the map $\left(f+\lambda \phi_{1}, \phi_{2}, \cdots, \phi_{k}\right)$ has rank $\geqq p$ at $x_{0}$.

Proof. We may suppose that $f\left(x_{0}\right)=\phi_{i}\left(x_{0}\right)=0$. Let $A$ be the analytic set of $x$ where $\phi_{2}(x)=\cdots=\phi_{k}(x)=0$. Let $A_{1}, \cdots, A_{m}$ be the irreducible components of $A$ containing $x_{0}$ and having dimension $>n-p$. The points of $A_{j}$ where $\phi_{1}(x)=0$ has dimension $\leqq n-p$ at $x_{0}$. Since the set of zeros of a nonconstant holomorphic function on an irreducible complex space of dimension $t$ is purely $t-1$ dimensional, $\operatorname{dim} A_{j}=n-p+1$. Clearly $\phi_{1} \neq 0$ on any $A_{j}$. If $f / \phi_{1}$ is nonconstant on some $A_{j}$ then $f+\lambda \phi_{1} \neq \equiv$ on $A_{j}$ for any $\lambda$. If $A_{1}, \cdots, A_{t}$ are those $A$ 's on which $f / \phi_{1}$ is constant and $f / \phi_{1}=\lambda_{j}$ on $A_{j}(j=1, \cdots, t)$, then $f+\lambda \phi_{1} \not \equiv 0$ on $A_{j}(j=1, \cdots, m)$ if $\lambda \neq-\lambda_{1}, \cdots,-\lambda_{t}$. Then, the set of points where $f+\lambda \phi_{1}=\phi_{2}=\cdots=\phi_{k}=0$ has dimension $\leqq n-p$ at $x_{0}$ and Lemma 2 follows.

Lemma 3. If for some $x_{0} \in X$ there is a map $f: X \rightarrow C^{k}$ of rank $\geqq p$ at $x_{0}$, then there is a map $\phi: X \rightarrow C^{p}$ of rank $=p$ at $x_{0}$ (and the components of $\phi$ may be chosen as linear combinations of those of $f$ ).

This is proved similarly to Lemma 2 .

Proof of Theorem 3. Let $R^{p}(X)$ be the space of $p$-tuples of holomorphic functions on $X$. If, for $x \in X, C(x)$ is the set of $f \in R^{p}(X)$ having rank $<p$ at $x$, then, by Lemma $1, C(x)$ is closed. By Lemmas $2,3, R^{p}(X)-C(x)$ is dense in $R^{p}(X)$ for if $f \in R^{p}(X)$ and $\left(\phi_{1}, \cdots, \phi_{p}\right)$ $\in R^{p}(X)-C(x), \lambda \neq 0$ then, there are $\mu_{i}, 0 \leqq \mu_{i} \leqq 1, i=1, \cdots, p$ such that $\left(f_{1}+\lambda \mu_{1} \phi_{1}, \cdots, f_{p}+\lambda \mu_{p} \phi_{p}\right)$ has rank $\geqq p$ at $x$, by Lemma 2 . It follows as in Theorem 2 that given a countable set $\left\{x_{m}\right\}$ on $X$, there is an $f \in R^{p}(X)$ of rank $\geqq p$ at $x_{m}$ for each $m$. Since, for any holomorphic map $\phi: X \rightarrow C^{k}$, the set of $x \in X$ with $r(\phi, x)<p$ is analytic in $X$ by [5, Satz 17], Theorem 3 follows if we apply downward induction on the dimension of analytic sets similar to the proof of Theorem 1.

It is clear, moreover, that we could require the mapping functions to approximate to arbitrarily given functions on a given compact set. 
Theorem 4. Let $X$ be a normal, connected complex space. Suppose that there exists a map $f=\left(f_{1}, \cdots, f_{k}\right): X \rightarrow C^{k}$ of constant rank $p$ on $X$. Then, to every $\epsilon>0$ and complex numbers $a_{\mu \nu}, \mu=1, \cdots, p, \nu=1, \cdots, k$, there exists a matrix $\left(b_{\mu \nu}\right)$ with $\left|b_{\mu \nu}-a_{\mu \nu}\right|<\epsilon$ such that the functions $\sum_{\nu=1}^{k} b_{\mu \nu} f_{\nu}, \mu=1, \cdots, p$ have rank $p$ at every point of $X$. In particular, there is a map $X \rightarrow C^{p}$ of rank $p$ at all points of $X$.

Proof. Let $\left(X^{*}, f^{*}\right)$ be a complex base of $(X, f)$ (see Stein [6]) and let $f=\Phi \circ f^{*}, \Phi=\left(\Phi_{1}, \cdots, \Phi_{k}\right): X^{*} \rightarrow C^{k}$. As in Lemma 3 , to any $x_{0} \in X$, there is a map $\left(\phi_{1}, \cdots, \phi_{p}\right): X \rightarrow C^{p}$ such that each $\phi_{i}$ is a linear combination of the $f_{j}$, such that $\phi_{1}, \cdots, \phi_{p}$ have rank $\geqq p$ at $x_{0}$ and so in a neighbourhood of $x_{0}$; since clearly $\phi_{i}$ "depend on $f$ " (in the sense of Stein), the $\phi_{i}$ have constant rank $p$ in an open neighbourhood $U$ of $x_{0}$ and so give an open map of $U$ onto an open set $V \supset\left(\phi_{1}\left(x_{0}\right), \cdots, \phi_{p}\left(x_{0}\right)\right) \in C^{p}$. Clearly $\phi_{i}=\psi_{i} \circ f^{*}$ where $\psi_{i}$ is a linear combination of $\Phi_{1}, \cdots, \Phi_{k}$. Clearly $\left(\psi_{1}, \cdots, \psi_{p}\right)$ give an open map of $f^{*}(U)$ onto $V$ and so they are nowhere degenerate in $f^{*}(U)$ of rank $p=\operatorname{dim} X^{*}$. Consequently, $\left(\Phi_{1}, \cdots, \Phi_{k}\right)$ is nowhere degenerate in $f^{*}(U)$. The result follows at once from [1, Satz 8 and Satz 11].

Theorem 4 is true even for arbitrary (non-normal) complex spaces $X$, if we suppose $X$ to be countable at infinity (or that $X$ has countably many irreducible components).

The conditions of Theorem 4 are satisfied if the following conditions are satisfied:

(a) $X$ is countable at infinity;

(b) for any $x_{0} \in X$, there is a map $f: X \rightarrow C^{p}$ with $r\left(f, x_{0}\right) \geqq p$;

(c) for any $x_{0} \in X$ and any map $\phi: X \rightarrow C^{p+1}$, we have $r\left(\phi, x_{0}\right) \leqq p$.

In this case, it is easily deduced from Theorems 3 and 4 that there is a map of $X$ in $C^{p}$ of rank $p$ on all $X$ which approximates to an arbitrarily given map of $X$ in $C^{p}$ on a given compact set.

Let now $X$ be a complex manifold and $f: X \rightarrow C^{k}$ a holomorphic map. We shall denote by $\rho\left(f, x_{0}\right)$, where $x_{0} \in X$, the rank at $x_{0}$ of the Jacobian matrix of $f$ with respect to any local coordinate system at $x_{0}$. Then we have

THEOREM 5. Let $X$ be countable at infinity and suppose that to any $x_{0} \in X$ there is a holomorphic map $f: X \rightarrow C^{k}$ with $\rho\left(f, x_{0}\right) \geqq p$. Then there is an integer $N=N(n)$ and a holomorphic map $\phi: X \rightarrow C^{N}$ with $\rho(\phi, x)$ $\geqq p$ for any $x \in X$.

Finally, we remark that the method used above can be used to prove 
THEOREM 6. Let $X$ be a complex space and suppose that there is an integer $N>0$ such that every $x_{0} \in X$ has a neighbourhood $U$ and $a$ map $f: X \rightarrow C^{N}$ which imbeds $U$ isomorphically onto an analytic set $A$ in an open set in $C^{N}$ (i.e. $f$ induces an isomorphism between the holomorphic functions on open sets in $U$ and functions on open sets of $A$ which are locally restrictions of holomorphic functions in open sets in $\left.C^{N}\right)$. Then there is a map $f: X \rightarrow C^{M}$ with this property at all points of $X$.

We construct a map $\phi: X \rightarrow C^{2 n+1}$ which is one-one in a neighbourhood of any point of $X$. (This follows similarly to Theorem 1 ). Then $\phi$ gives a one-one proper map of a neighbourhood $U$ of any point of $X$ into a sphere $H$ in $C^{2 n+1}$. The set of points where $\phi$ fails to have the property of Theorem 6 can then be shown to be an analytic set as follows. If $X$ is normal, this follows at once from the theorem of $\mathrm{K}$. Oka that the set of non-normal points of an analytic set is itself analytic [3]. In the general case $\phi(U)$ is analytic in $H$. If $D$ is the structure sheaf of $U$ and $\mathfrak{F}$ that of $\phi(U)$, then $\phi_{0}(\mathfrak{D})$ the 0th image of $\mathfrak{O}$ (see [2]) is a coherent sheaf on $H$ containing $\mathfrak{F}$ (see [2, Satz 27]). Hence $\phi_{0}(D) / \mathfrak{F}$ is a coherent sheaf, and the set of exceptional points in $U$ is the inverse image of the set of points $a$ where $\left(\phi_{0}(\mathfrak{O}) / \mathfrak{F}\right)_{a} \neq 0$, which is analytic. We can prove, analogously to the proof of Theorem 3 , that the set of $f \in R^{N}(X)$ such that $(\phi, f)$ fails to imbed any neighbourhood of a given point $x_{0} \in X$ isomorphically is nowhere dense in $R^{N}(X)$ (see proof of [1, Satz 3]). Theorem 6 follows as before.

Remmert [4] has proved that any holomorphically complete normal space has a one-one proper imbedding in some $C^{N}$. It follows from Theorem 6, that if in addition, we suppose that any point of $X$ has a neighbourhood which has a normal imbedding in an open set of $C^{k}$ where $k$ is bounded on $X$, then $X$ is isomorphic to a normal analytic set in some $C^{M}$.

\section{BIBLIOGRAPHY}

1. H. Grauert, Charakterisierung der holomorph-vollständigen komplexen Räume, Math. Ann. vol. 129 (1955) pp. 233-259.

2. H. Grauert and R. Remmert, Komplexe Räume, Math. Ann. vol. 136 (1958) pp. 245-318.

3. K. Oka, Sur les fonctions analytiques de plusieurs variables, VIII. Lemme fondamental, J. Math. Soc. Japan vol. 3 (1951) pp. 204-214, 259-278.

4. R. Remmert, Sur les espaces holomorphiquement séparables et holomorphiquement convexes, C. R. Acad. Sci. Paris vol. 243 (1956) pp. 118-121.

5. - Holomorphe und meromorphe Abbildungen komplexer Räume, Math. Ann. vol. 133 (1957) pp. 328-370.

6. K. Stein, Die Existenz komplexer Basen zu holomorphen Abbildungen, Math. Ann. vol. 136 (1958) pp. 1-8.

Tata Institute of Fundamental Research, Bombay, India 